\title{
Random-neighbor Olami-Feder-Christensen slip-stick model
}

\author{
Osame Kinouchi, ${ }^{*}$ Suani T. R. Pinho, ${ }^{\dagger}$ and Carmem P. C. Prado \\ Departamento de Física Geral, Instituto de Física, Universidade de São Paulo, Caixa Postal 66318, \\ CEP 05315-970 São Paulo, São Paulo, Brazil
}

(Received 24 March 1998)

\begin{abstract}
We reconsider the treatment of Lise and Jensen [Phys. Rev. Lett. 76, 2326 (1996)] on the random neighbor Olami-Feder-Christensen stik-slip model [Phys. Rev. Lett. 68, 1244 (1992)] and examine the strong dependence of the results on the approximations used for the distribution of states $p(E)$. [S1063-651X(98)09308-8]
\end{abstract}

PACS number(s): 05.40.+j, 05.70.Jk, 05.70.Ln

The work of Olami, Feder, and Christensen [1] on a slipstick earthquake model indicated, some time ago, that selforganized criticality (SOC) may occur without a local conservation law. Recently, it has been claimed by Lise and Jensen [2] that the random-neighbor version of the OlamiFeder-Christensen (OFC) model also presents critical behavior above some critical dissipation level $\alpha_{c}<\alpha_{0}$, where $\alpha_{0}$ $=1 / q$ is associated with local conservation for $q$ neighbors. These authors based their claims on some theoretical meanfield arguments and on numerical simulations with systems with up to $N=400^{2}$ sites. In order to perform the mean-field calculation they had to make many different assumptions about the behavior of the model.

More recently, Chabanol and Hakin [3] and Bröker and Grassberger [4] performed a more detailed analysis of the same model, showing that what has been interpreted as a critical behavior in [2] indeed corresponds to a subcritical region with very large (but finite) mean avalanche sizes. Although Bröker and Grassberger [4] give a comprehensive treatment of the random-neighbor version of the OFC model (which we will designate ROFC), it may be of interest to detect exactly where the theoretical arguments given in [2] fail, since that point is not transparent in their paper and similar problems may occur or be of interest in the future. This is the aim of our paper. We will show that the problem is not in the method used in [2] (which eventually can give useful information about the mechanism behind SOC) but in the strong dependence of the output of the calculations on the exact form of the distribution of states $p(E)$ of the system.

To reinforce the strong dependence of the results on the specific form of $p(E)$, we revisit the ROFC model, but this time introducing a simple and small modification on the $p(E)$ distribution, which consists in replacing the interval $\left[0, E_{c}\right]$ where the uniform distribution used by Lise and Jensen was defined by the interval $\left[0, E^{\star}\right]$, with $E^{\star}$ $<E_{c}$ ( $E_{c}$ is the threshold value above which the sites become unstable and relax), that is,

$$
p(E)=\frac{1}{E^{\star}} \Theta(E) \Theta\left(E^{\star}-E\right),
$$

\footnotetext{
*Electronic address: osame@ultra3000.ifqsc.sc.usp.br

${ }^{\dagger}$ On leave from the Instituto de Física, Universidade Federal de

Bahia. Electronic address: spinho@gibbs.if.usp.br

†Electronic address: prado@if.usp.br
}

where $\Theta(x)$ is the Heaviside function [see Fig. 1(a)].

The random version of the OFC model (ROFC) consists of $N$ sites initially with an energy $E_{i}<E_{c}$, for $i=1, \ldots, N$. The sites with energy $E$ below $E_{c}$ are stable sites (inactive) and will be labeled by a minus superscript; the sites with energy $E$ above $E_{c}$ are unstable (active) and will be labeled by a plus superscript. The energies of all sites are increased slowly until the instant $t$ when the energy of a certain site $i$ reaches the value $E_{c}$. This site then becomes unstable and the system relaxes in a very short time scale according to the rules

$$
E_{i}(t+1)=0, \quad E_{r n}(t+1)=E_{r n}(t)+\alpha E^{+},
$$

where $E_{r n}$ stands for the energy of $q$ other sites chosen at random and $\alpha \leqslant 1 / q$. Eventually some of these $q$ sites may become unstable and also relax and so may generate an avalanching process that stops only when the energies of all sites are again below $E_{c}$. If we have $\alpha=\alpha_{c}=1 / q$ we say that the system is conservative.

Following [2], the probability of an inactive site to be activated by receiving a contribution $\alpha E^{+}$of an active site is

$$
P_{+}\left(E^{+}\right)=\frac{\int_{E_{c}-\alpha E^{+}}^{E_{c}} p(E) d E}{\int_{0}^{\infty} p(E) d E}=\frac{E^{\star}-E_{c}+\alpha E^{+}}{E^{\star}} .
$$

The branching ratio $\sigma$ is the average number of new unstable sites created by an unstable site that relaxes. Clearly, in order to have a critical branching process, we must have $\sigma=1$. For $q$ random neighbors we have

$$
\sigma=q \frac{\int_{E_{c}}^{\infty} d E^{+} P_{+}\left(E^{+}\right) p\left(E^{+}\right)}{\int_{E_{c}}^{\infty} d E^{+} p\left(E^{+}\right)},
$$

where $p\left(E^{+}\right)$is the distribution of states for unstable sites, that is, sites with energy above $E^{\star}$. Adopting the notation $\langle\cdots\rangle \equiv \int(\cdots) p(E) d E / \int p(E) d E$ for averages, we find

$$
\sigma=q\left[\frac{E^{\star}-E_{c}}{E^{\star}}+\frac{\alpha}{E^{\star}}\left\langle E^{+}\right\rangle\right] .
$$



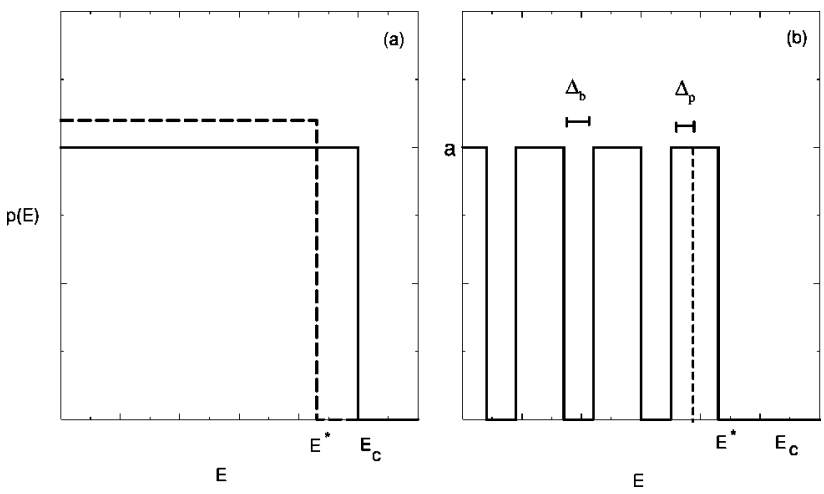

FIG. 1. (a) Uniform approximation for the energy distribution $p(E)$ of the ROFC model. The solid line corresponds to the interval of $E$ used by Lise and Jensen, [0, $\left.E_{c}\right]$; the dashed line corresponds to a smaller interval of $E,\left[0, E^{\star}\right]$, used in our calculations. (b) A more realistic approximation (non-uniform) of the energy distribution of the ROFC model, for $q=4$ (with four peaks). The parameters $\Delta_{p}$ and $\Delta_{b}$ are, respectively, the half-width of the peaks and the width of the gaps. The value of $p(E)$ at the peaks is $a$ and $E_{c}$ $\geqslant E^{\star}=7 \Delta_{p}+3 \Delta_{b}$.

The critical branching ratio $\sigma=1$ defines a value $\alpha_{c}$ above which infinite avalanches may occur. The quantity $\left\langle E^{+}\right\rangle$is estimated [2] as

$$
\left\langle E^{+}\right\rangle=\frac{\left\langle E^{-}\right\rangle}{1-\alpha} .
$$

The average on $E^{-}$is calculated as

$$
\begin{aligned}
\left\langle E^{-}\right\rangle & =\frac{\int_{E_{c}-\alpha E^{+}}^{E_{c}} p(E) d E}{\int_{E_{c}-\alpha E^{+}}^{E_{c}} p(E) d E} \\
& =\frac{1}{E^{\star}-E_{c}+\alpha\left\langle E^{+}\right\rangle} \int_{E_{c}-\alpha\left\langle E^{+}\right\rangle}^{E^{\star}} E^{-} d E \\
& =\frac{1}{2} \frac{\left(E^{\star}\right)^{2}-E_{c}^{2}+\left(2 E_{c}-\alpha\left\langle E^{+}\right\rangle\right) \alpha\left\langle E^{+}\right\rangle}{E^{\star}-E_{c}+\alpha\left\langle E^{+}\right\rangle} .
\end{aligned}
$$

With this result and Eq. (6) we get

$$
\left(2 \alpha-\alpha^{2}\right) x^{2}+\left[2 E^{\star}(1-\alpha)-2 E_{c}\right] x+E_{c}^{2}-\left(E^{\star}\right)^{2}=0,
$$

where $x=\left\langle E^{+}\right\rangle$. This leads to the simple solution

$$
\left\langle E^{+}\right\rangle=\frac{E_{c}+E^{\star}}{2-\alpha} .
$$

Finally, we get for the branching ratio

$$
\sigma=q\left[\frac{E^{\star}-E_{c}}{E^{\star}}+\frac{\alpha\left(E_{c}+E^{\star}\right)}{E^{\star}(2-\alpha)}\right] .
$$

The critical condition $\sigma=1$ leads to

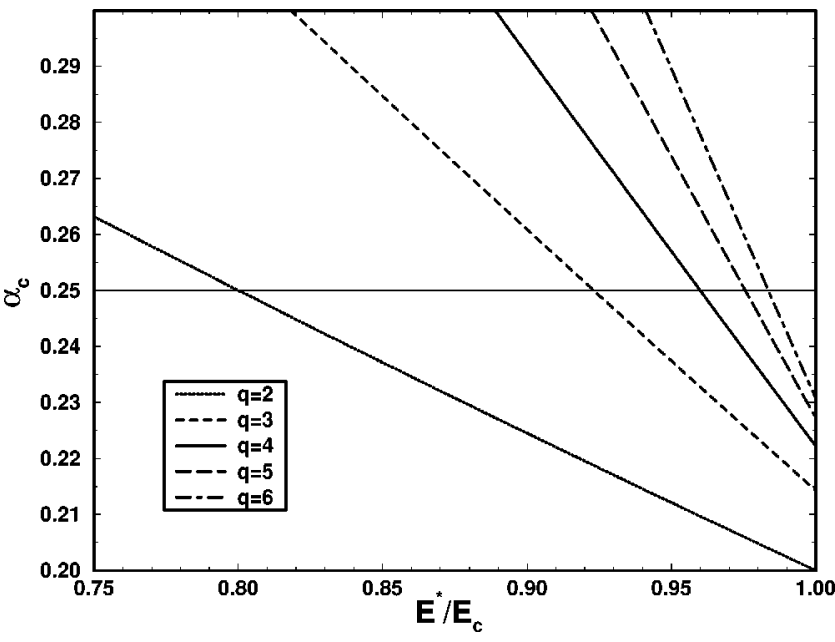

FIG. 2. Normalized "critical" dissipation level $\tilde{\alpha}_{c}=k \alpha / 4$ as a function of $E^{\star}$. Values for $\tilde{\alpha}_{c}$ above 0.25 are not physically admissible.

$$
\alpha_{c}=\frac{E_{c}-E^{\star}(q-1) / q}{E_{c}+E^{\star} / 2 q} .
$$

For example, if $q=4$ (the case studied in [2]), we have

$$
\alpha_{c}=\frac{E_{c}-3 E^{\star} / 4}{E_{c}+E^{\star} / 8} .
$$

If we consider $E^{\star}=E_{c}$ we recover Lise and Jensen's value $\alpha_{c}=2 / 9$. However, the value of $\alpha_{c}$ has a strong dependence on the value of $E^{\star}$. We see that, already for the value $E^{\star} / E_{c}=24 / 25=0.96, \alpha_{c}$ achieves the physical limit 0.25 .

In Fig. 2 we show the behavior of the normalized coupling $\tilde{\alpha}_{c}=q \alpha_{c} / 4$ as a function of $E^{\star}$. With this normalization, the conservative case always corresponds to $\tilde{\alpha}=0.25$ (as for $q=4$ ). We see that the allowed region of values for $E^{\star}$ so that $\tilde{\alpha}_{c}<0.25$ is very narrow for any $q$ and that the value of $\alpha_{c}$ varies strongly in this region. We have already shown in another work (see [5]) that already with lattices with $N=600^{2}$ sites it is possible to see a finite mean size avalanche for $\alpha=0.23$, in contrast to the results of [2] based on simulations in lattices with $N=400^{2}$.

Besides showing that Lise and Jensen's approach is not robust with respect to $p(E)$, we may ask what kind of model produces the uniform distribution

$$
p(E)=\Theta(E) \Theta(1-E)
$$

used by those authors. We found that an extremal version of the Feder-Feder model, hereforth called the EFF model, indeed produces this distribution. Extremal here is used in the same sense it was used in the Bak-Snappen coevolution model [6]. In the dynamics of extremal models there is no driving step. We locate the site $i$ with the largest value of $E_{i}=\max \left\{E_{j}\right\}$ at the initial instant $t$. This site relaxes following the original Feder-Feder rules

$$
E_{i}^{m}(t+1)=\eta, \quad E_{n n}(t+1)=E_{n n}(t)+\alpha,
$$



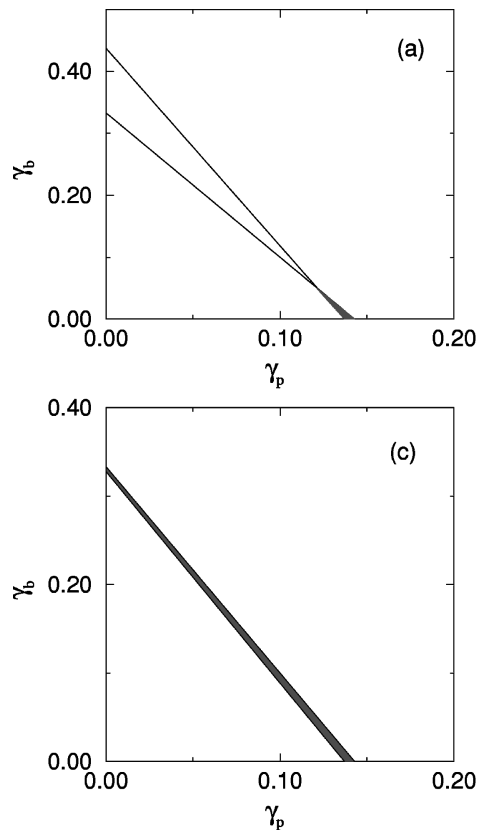
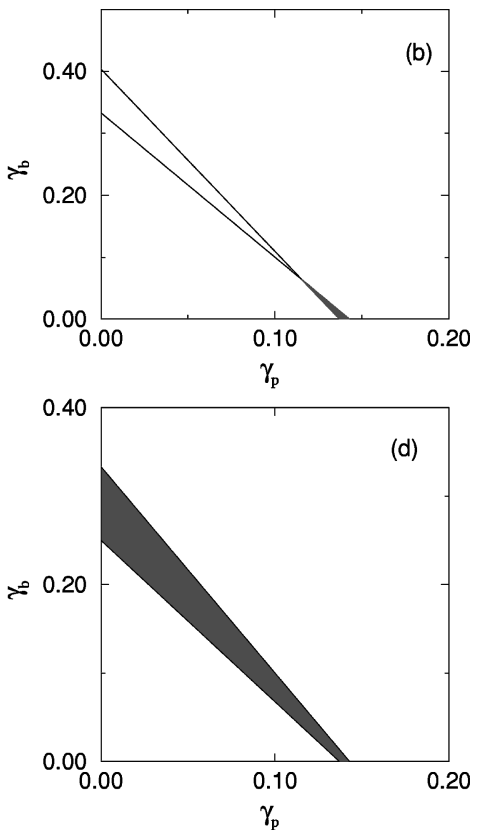

FIG. 3. Space of parameters for $p(E)$ in terms of $\gamma_{p}=\Delta_{p} / E_{c}$ and $\gamma_{b}=\Delta_{b} / E_{c}$. The shaded regions correspond to the intersection between $\alpha$ $\leqslant 1 / 4$ and $\left(7 \Delta_{p}+3 \Delta_{b}\right) / E_{c}=7 \gamma_{p}+3 \gamma_{b} \leqslant 1$. Depending on the value of $E_{c}-\alpha E^{+}$, we have (a) $E_{c}-\alpha E^{+} \epsilon\left[0, \Delta_{p}\right] ;$ (b) $E_{c}-\alpha E^{+} \epsilon\left[\Delta_{p}+\Delta_{b}, 3 \Delta_{p}\right.$ $\left.+\Delta_{b}\right]$, (c) $E_{c}-\alpha E^{+} \epsilon\left[3 \Delta_{p}+2 \Delta_{b}, 5 \Delta_{p}+2 \Delta_{b}\right]$, and (d) $E_{c}-\alpha E^{+} \epsilon\left[5 \Delta_{p}+3 \Delta_{b}, 7 \Delta_{p}+3 \Delta_{b}\right]$. where $\eta$ is a noise, $\eta \in[0, \epsilon]$. If we consider $\epsilon=\alpha=1 / 4$ we will have Eq. (13). In this model the size of an avalanche is defined as the number of sites with energy $E_{i}^{m}>1$ that relax in a row. Now, if we repeat Lise and Jensen's calculation using these EFF rules instead of the OFC rules, we get the self-consistent result $\alpha_{c}=E_{c} / q$.

It is also possible to show that a more realistic assumption about $p(E)$ leads essentially to the same results obtained by $[3,4]$. If we simulate the ROFC model with $q=4$ we will get an energy distribution $p(E)$ with four peaks [2]. They show clearly that $p(E)$ is not a simple constant. We then decided to repeat the same calculations but supposing this time that $p(E)$ had the (more realistic) form shown in Fig. 1(b), where $\Delta_{p}$ is half the width of each peak and $\Delta_{b}$ is the width of the gaps between two peaks. This means that

$p(E)= \begin{cases}a & \text { for } \quad E \in I_{1}, \quad E \in I_{2}, \quad E \in I_{3}, \quad \text { or } E \in I_{4} \\ 0 & \text { otherwise, }\end{cases}$

where $I_{1}=\left[0, \Delta_{p}\right], \quad I_{2}=\left[\Delta_{p}+\Delta_{b}, 3 \Delta_{p}+\Delta_{b}\right], \quad I_{3}=\left[3 \Delta_{p}\right.$ $\left.+2 \Delta_{b}, 5 \Delta_{p}+2 \Delta_{b}\right]$, and $I_{4}=\left[5 \Delta_{p}+3 \Delta_{b}, 7 \Delta_{p}+3 \Delta_{b}\right]$.

We also have that $E^{*}=3 \Delta_{b}+7 \Delta_{p}$ is the maximum value for which $p(E) \neq 0$. Then we have

$$
P_{+}\left(E^{+}\right)=\frac{\int_{E_{c}-\alpha E^{+}}^{E_{c}} p(E) d E}{\int_{0}^{\infty} p(E) d E}=\frac{1}{7 a \Delta_{p}} .
$$

The lower limit of the integral in the numerator $E_{c}$ $-\alpha E^{+}$can now belong to any of the four intervals that define the peaks of the distribution, to which we will asssign the indices $i=1,2,3,4$. Considering each one of the possibilities, the integrals $P_{+}^{i}\left(E^{+}\right)$assume the generic form

$$
P_{+}^{i}\left(E^{+}\right)=1+\frac{(i-1) \Delta_{b}}{7 \Delta_{p}}-\frac{E_{c}}{7 \Delta_{p}}+\frac{\alpha E^{+}}{7 \Delta_{p}}
$$

The branching rate is given by

$$
\sigma=4 P_{+}^{i}=4\left[1+\frac{(i-1) \Delta_{b}}{7 \Delta_{p}}-\frac{E_{c}}{7 \Delta_{p}}+\frac{\alpha\left\langle E^{+}\right\rangle}{7 \Delta_{p}}\right] .
$$

In a similar way used to obtain Eq. (7), we calculate an expression for $\left\langle E^{-}\right\rangle$, which is associated with Eq. (6), which leads

$$
\begin{aligned}
\left\langle E^{+}\right\rangle^{i}= & \frac{E_{c}}{\alpha(2-\alpha)}-\frac{\left[7 \Delta_{p}+(i-1) \Delta_{b}\right](1-\alpha)}{\alpha(2-\alpha)} \\
& \pm \frac{\sqrt{y_{i}}}{2 \alpha(2-\alpha)},
\end{aligned}
$$

where

$$
\begin{aligned}
y_{i}= & 4\left\{E_{c}(1-\alpha)-\left[7 \Delta_{p}+(i-1) \Delta_{b}\right]\right\}^{2} \\
& +4 \alpha(2-\alpha)\left[x_{i}-14(i-1)\right] \Delta_{p} \Delta_{b},
\end{aligned}
$$

with $x_{i}=24,26,32$, and 42, for $i=1,2,3$, and 4, respectively. Imposing the branching condition $\sigma=1$ and using Eq. (19) we get

$$
7 \Delta_{p}(2+\alpha)+4(i-1) \Delta_{b}-4 E_{c}(1-\alpha)+7 \alpha E_{c} \pm \sqrt{y_{i}}=0 .
$$

For instance, if we take $\Delta_{p}=0.08$ and $\Delta_{b}=0.1$ [which corresponds to Fig. 1(b)], the critical branching condition leads to values of $\alpha^{*}$ outside the physical range (that is, $\alpha^{\star}$ $>1 / 4$ ). Therefore, in this particular example, it is physically forbidden to assume that $\sigma=1$, so there is no self-organized critical state.

If we take the limit for the conservative case (that is, $\Delta_{p}$ $\rightarrow 0$ and $\Delta_{b} \rightarrow \alpha E_{c}$ ), the four peaks tend to four $\delta$ functions at $(i-1) \alpha E_{c}$ and it is easy to see that the condition $\sigma=1$ leads to the only possibility $\alpha^{*}=\alpha_{c}=1 / 4$ (we obtain $\alpha^{*}$ $>1 / 4$ for $i=1,2,3)$. It can also be shown that if we consider 
the limit $\Delta_{b} \rightarrow 0$ and $\Delta_{p} \rightarrow E_{c} / 7$ ( that is, $p(E)$ is constant in the interval $\left[0, E_{c}\right]$, which corresponds to the approximation of Ref. [2]), then $\alpha^{*}=2 / 9$.

In general, for all values of $i$, the regions of the parameter space associated with $\alpha \leqslant 1 / 4$ are determined by

$$
E_{c}-\frac{175}{24} \Delta_{p}-\frac{2 x_{i}}{21} \Delta_{b} \leqslant 0 .
$$

From this inequality plus the relation $E_{c} \geqslant 7 \Delta_{p}+3 \Delta_{b}$ (see Fig. 3) we see that only for a very small range of the parameters $\Delta_{p}$ and $\Delta_{b}$ are there values of $\alpha_{c}$ in the physical range $\left(0<\alpha_{c} \leqslant 1 / 4\right)$. In all of those cases, $\Delta_{b}$ is very small and the shape of $p(E)$ is very close to the constant form used by Lise and Jensen. Moreover, $\alpha$ varies strongly in these allowed ranges.

In conclusion, we showed that, besides not having considered lattices big enough, the problem with the approach used by Lise and Jensen in [2] is not in the method itself, but in the strong dependence of the output of the calculations on the compatibility between the distribution $p(E)$ and the assumed dynamical rules that presumably lead to it. We also showed that the $p(E)$ approximation used by Lise and Jensen does not correspond to the model they intend to analyze, namely ROFC, but to another nonconservative model, the extremal Feder-Feder model. If we adopt the EFF dynamical rules, Lise and Jensen's method will lead to the right conclusions. In the end, we followed the same approach but now consider a more realistic approach for $p(E)$ and get essentially the same results that had already been obtained through the use of other arguments in [3,4], that is, in the ROFC model there is SOC only in the conservative limit.

We are thankful to S. R. Salinas for useful discussions about the analytical results of the R-OFC model. S. T. R. P. acknowledges the support by the Brazilian agency CAPESPICD.
[1] Z. Olami, H. J. F. Feder, and K. Christensen, Phys. Rev. Lett. 68, 1244 (1992).

[2] S. Lise and H. J. Jensen, Phys. Rev. Lett. 76, 2326 (1996).

[3] M-L. Chabanol and V. Hakin, Phys. Rev. E 56, R2343 (1997).
[4] H.-M. Bröker and P. Grassberger, Phys. Rev. E 56, 3944 (1997).

[5] S. T. R. Pinho, C. P. C. Prado, and O. Kinouchi, Physica A (to be published).

[6] P. Bak and K. Sneppen, Phys. Rev. Lett. 71, 4083 (1993). 